Radiologe $2017 \cdot 57: 840-849$

DOI 10.1007/s00117-017-0285-0

Online publiziert: 21. Juli 2017

(c) Der/die Autor(en) 2017. Dieser Artikel ist

eine Open-Access-Publikation.

CrossMark

G. Widmann ${ }^{1}$ - V. A. Nguyen ${ }^{2} \cdot$ J. Plaickner ${ }^{1} \cdot$ W. Jaschke ${ }^{1}$

'Universitätsklinik für Radiologie, Medizinische Universität Innsbruck, Innsbruck, Österreich

${ }^{2}$ Universitätsklinik für Dermatologie, Venerologie und Allergologie, Medizinische Universität Innsbruck, Innsbruck, Österreich

\title{
Unerwünschte Wirkungen der Immuntherapie
}

\section{Klinik, radiologische und nuklearmedizinische Befunde}

tion und Funktion der T-Zellen. Nivolumab und Pembrolizumab blockieren die Interaktion zwischen dem Cell-deathprotein-1(PD-1)-Rezeptor an T-Zellen und seinen Liganden PD-L1 und PD-L2, an Antigen-präsentierenden Zellen und Tumorzellen. Im Unterschied zur CTLA4-Inhibition initiieren PD-1-Inhibitoren somit auch eine tumorspezifische Interaktion mit dem Immunsystem.

Als Folge der reduzierten Selbsttoleranz können Immuncheckpointinhibitoren ein breites Spektrum immunbedingter Nebenwirkungen auslösen, die jedes Organ und System betreffen können [12, 26].

\section{Allgemeine klinische Aspekte}

Die Inzidenz von Nebenwirkungen der Immuncheckpointinhibitoren ist hoch und betrifft bis $\mathrm{zu} 80-90 \%$ der Patienten [18]. Symptomatische immunbedingte Nebenwirkungen zeigen ein breites klinisches Spektrum und werden nach den Common Terminology Criteria for Adverse Events (CTCAE) als Grad 1 (mild), Grad 2 (moderat), Grad 3 (schwer) und Grad 4 (lebensbedrohlich) eingeteilt. Ein aktueller systematischer Übersichtsartikel von publizierten Toxizitäten aus Phase-II- und -III-Studien beziffert die Häufigkeiten von immunbedingten Nebenwirkungen Grad 3 und 4 mit 20-30\% für Ipilimumab, 10-15\% für Nivolumab und Pembrolizumab, und $55 \%$ für die Kombinationstherapie Ipilimumab und Nivolumab [26]. Toxizitäten von Ipilimumab sind dosis- abhängig und zeigen einer höhere Rate gastrointestinaler, dermatologischer und endokriner Nebenwirkungen [19, 26]. Im Gegensatz dazu sind die Toxizitäten von Nivolumab und Pembrolizumab dosisunabhängig und mit höheren Raten von hepatologischen und pulmonalen Nebenwirkungen assoziiert $[19,26]$. Der Beginn immunbedingter Nebenwirkungen nach Therapiestart von Ipilimumab zeigt eine zeitliche Assoziation [19]:

- Hautmanifestationen nach 2 bis

3 Wochen (1. Dosis),

- Kolitis nach 5 bis 10 Wochen (2. Dosis),

- Hepatitis nach 12 bis 16 Wochen

(3. Dosis),

- endokrine Dysfunktionen nach

9 Wochen (4. Dosis) und

- Pneumonitis nach 8 bis 14 Wochen.

Manche Patienten weisen simultan mehre als eine Nebenwirkung auf [7].

Das Management der immunbedingten Nebenwirkungen richtet sich nach der klinischen Einschätzung und folgt den CTCAE-Richtlinien [19, 26]. Als generelle Behandlungsstrategie wird bei Grad-2-Toxizitäten die Behandlung mit Immuncheckpointinhibitoren unterbrochen und erst dann wieder begonnen, wenn die Symptome unter Grad 1 zurückgehen. Die Behandlung von Grad3- und -4-Toxizitäten erfordert eine Hochdosistherapie mit Glukokortikoiden, eventuell unterstützt mit zusätzlicher Gabe von Immunsuppressiva, und einen permanenten Abbruch der Immuncheckpoint-Inhibitortherapie bei 
Hier steht eine Anzeige.

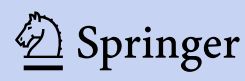


Patienten mit Grad-4-Toxizität. Mit der prompten Initiierung einer adäquaten Therapie lassen sich die meisten immunbedingten Nebenwirkungen erfolgreich behandeln.

\section{Radiologische und nuklear- medizinische Manifestationen}

Mit bildgebenden Verfahren darstellbare Manifestationen der immunbedingten Nebenwirkungen von Checkpointinhibitoren umfassen Kolitis, Hepatitis, Pankreatitis, Hypophysitis, Thyreoiditis, Pneumonitis, Arthritis, sarkoidähnliche Lymphadenopathie und entzündliche Veränderungen der Weichteile (Myositis, Fasziitis und Dichteerhöhungen des retroperitonealen Fettgewebes [5, 6, 28]). Publizierte Inzidenzen radiologischer Auffälligkeiten aus retrospektiven Studien von Patienten und Patientinnen mit metastasiertem Melanom, die mit Ipilimumab behandelt wurden, erreichen bis zu $31 \%[6,28]$. Es scheint einen direkten Zusammenhang zwischen der Inzidenz immunbedingter Nebenwirkungen und dem klinischen Response zu geben [6]. Nach Behandlung der immunbedingten Nebenwirkungen zeigen bis $\mathrm{zu} 90 \%$ der radiologischen und nuklearmedizinischen Befunde einen vollständigen Rückgang [6, 28].

\section{Kolitis}

Neben dermatologischen Manifestation wie Juckreiz und Hautausschlag zählen gastrointestinale Toxizitäten zu den häufigsten immunbedingten Nebenwirkungen [12]. Diarrhö und Kolitis treten typischerweise 5 Wochen nach Therapiebeginn auf und weisen eine Prävalenz von $36-38 \%$ und $8-10 \%$ für Ipilimumab bzw. $8-20 \%$ und 1-3 \% für die Gruppe Nivolumab und Pembrolizumab auf [19]. Eine Kolitis Grad 3 und 4 findet man in 7-9\% für Ipilimumab und in ungefähr 1-2\% für Nivolumab oder Pembrolizumab [19, 26]. Schwere und lebensbedrohliche Ereignisse resultieren aus Durchfall mit signifikanten Blutungen und intestinaler Perforation [9, 12, 17].

In einer retrospektiven Fallserie mit 16 Patienten mit Ipilimumab-assoziierter Kolitis und verfügbarer CT-Un-

Radiologe 2017· 57:840-849 DOI 10.1007/s00117-017-0285-0

(c) Der/die Autor(en) 2017. Dieser Artikel ist eine Open-Access-Publikation.

\section{G. Widmann · V. A. Nguyen · J. Plaickner · W. Jaschke}

\section{Unerwünschte Wirkungen der Immuntherapie. Klinik, radiologische und nuklearmedizinische Befunde}

\section{Zusammenfassung}

Hintergrund. Mit zunehmendem Einsatz von Immuntherapien in der Onkologie steigt die Bedeutung der radiologischen Beurteilung häufiger und schwerwiegender immunbedingter Nebenwirkungen.

Fragestellung. Inzidenz und Manifestation radiologischer und nuklearmedizinischer Befunde der immunbedingten Nebenwirkungen.

Material und Methode. Literaturübersicht zu Klinik und bildgebenden Befunden immunbedingter Nebenwirkungen der Immuncheckpointinhibitoren Ipilimumab, Nivolumab und Pembrolizumab. Veranschaulichung mit Bildbeispielen und Gegenübergestellung mit anderen relevanten Differenzialdiagnosen.

Ergebnisse. Die häufigsten bildgebenden Manifestationen sind Kolitis, Hepatitis, Pankreatitis, Hypophysitis, Pneumonitis, Arthritis und die sarkoidähnliche Lymphadenopathie. Schwere bis lebensbedrohliche Komplikationen können aus einer Kolitis, Pneumonitis und Hypophysitis resultieren. Eine klare Abgrenzung zu Autoimmunerkrankungen sowie Differenzierung von immunbedingten und entzündlichen pulmonalen Veränderungen kann sehr schwierig sein und bedarf einer engen multidisziplinären Zusammenarbeit. Schlussfolgerungen. Das Wissen um die klinischen und typischen bildgebenden Befunde von Nebenwirkungen der Immuntherapie ist essenziell, um rasche und adäquate Therapieentscheidungen treffen zu können. Neben Staging und Verlaufsbeurteilung ergänzt das Erkennen und Kontrollieren therapieassoziierter Nebenwirkungen die Verantwortung der Radiologie bei der Behandlung onkologischer Patienten.

\section{Schlüsselwörter}

Immuncheckpointinhibitoren · Immunbedingte Nebenwirkungen - Bildgebende Befunde - Computertomographie . Magnetresonanztomographie

\section{Adverse effects of immunotherapy. Clinical aspects, radiological and nuclear medicine results}

\section{Abstract}

Background. The increasing use of immunotherapy in oncology increases the need for radiologic evaluation of frequent and severe immune-related adverse events.

Objective. Determination of the incidence and manifestation of radiologic and nuclear medicine findings of immune-related adverse events.

Material and methods. Literature review of clinical and imaging findings of immunerelated adverse events induced by the immune checkpoint inhibitors ipilimumab, nivolumab and pembrolizumab. Findings are illustrated with pictorial examples and contrasted to other relevant differential diagnoses.

Results. The most frequent imaging manifestations are colitis, hepatitis, pancreatitis, hypophysitis, pneumonitis, arthritis and sarcoid-like lymphadenopathy. Severe to life-threatening complications may result from colitis, pneumonitis and hypophysitis. A clear differentiation from other autoimmune diseases and discrimination of immunerelated and infectious pulmonary findings can be very difficult and need close multidisciplinary collaboration. Conclusion. Knowledge of clinical and imaging findings of adverse events induced by immunotherapy is essential for timely and adequate therapeutic decisions. In addition to staging and follow-up imaging, identification and monitoring of immune-related adverse events adds to the radiologic responsibility in oncologic care.

\section{Keywords}

Immune checkpoint inhibitors - Immunerelated adverse events - Imaging results . Computed tomography - Magnetic resonance tomography 


\section{Immunbedingte}

Nebenwirkung

Kolitis

Hepatitis

Pankreatitis

Pneumonitis

Hypophysitis

Arthritis
Typische bildgebende Befunde

\section{Muster:}

Diffuse Kolitis (diffuse Wandverdickung, vermehrte Kontrastmittelaufnahme der Mukosa, vermehrte mesenteriale Gefäßzeichnung)

Segmentale Kolitis assoziiert mit Divertikulitis (Wandverdickung, perikolische Fettgewebsdichteerhöhung, vermehrte mesenteriale Gefäßzeichnung, vermehrte Kontrastmittelaufnahme der Mukosa, Divertikel) Isolierte rektosigmoidale Kolitis ohne Divertikulose (Wandverdickung, vermehrte Kontrastmittelaufnahme der Mukosa, perikolische Fettgewebsdichteerhöhung)

CT, MRT: Hepatomegalie, periportales Ödem, verminderte Leberdichte im Vergleich zur Baseline-CT, periportale T2-Signalsteigerung, vergrößerte periportale Lymphknoten

Ultraschall: periportale Echogenitätssteigerung und Gallenblasenwandödem

CT, MRT: Organvergrößerung, Verminderung der Parenchymdichte, umgebende Fettgewebsdichteerhöhung PET-CT: vermehrte FDG-Aufnahme

\section{Muster:}

kryptogene organisierende Pneumonie (COP) Nichtspezifische interstitielle Pneumonie (NSIP)

Hypersensitivitätspneumonitis (HP) und akute interstitielle Pneumonie/akutes respiratorisches Distresssyndrom (AIP/ARDS)

Befall:

untere $>$ mittlere $>$ obere Lungenabschnitte Verteilung:

gemischt und multifokal > peripher und basal Spezifische Befunde:

Mattglasverschattungen, retikuläre Verschattungen, Konsolidierungen

Moderate Vergrößerung der Hypophyse, konvexe Kontur, Vergrößerung des Stiels oder Infundibulums, homogene Kontrastmittelaufnahme

Ultraschall und MRT: proliferative Synovitis mit typischer Hyperämie und synovialer Verdickung, Gelenkerguss, manchmal Gelenkerosionen, Tenosynovitis, Knochenmarködem

PET-CT: erhöhte FDG-Aufnahme in der Synovia multipler bilateraler Gelenke

Muster:

unspezifisch, große und/oder kleine Gelenke, Oligoarthritis, additive Arthritis, oder schwere Polyarthritis
Wichtige Differenzialdiagnosen

\section{Morbus Crohn:}

Involviert vorwiegend das terminale lleum, ungleichmäßige und transmurale, entzündliche Dichteerhöhung des umliegenden Fettgewebes, submukosales Fett, "fibrofatty hyperplasia", Fisteln Colitis ulcerosa:

Befällt typischerweise das Kolon mit zunehmender Intensität nach distal, diffuse Atrophie der Krypten, submuköses Halo oder Fett Infektiöse Kolitis:

Vorwiegend limitiert auf das rechte Kolon, Wandverdickung, homogene Kontrastmittelaufnahme

Pseudomembranöse Kolitis:

Ausgeprägte zirkumferenzielle oder exzentrische Wandverdickung des gesamten Kolons

Virale Hepatitis:

positive Virusserologie

Akute alkoholtoxische Lebererkrankung:

Alkoholmissbrauch, Steatohepatitis, Zirrhosis hepatis

Idiopathische Autoimmunhepatitis:

Autoantikörper, Zirrhose

Immunoglobulin-G4-assoziierte Autoimmunpankreatitis:

Auflösung der normalen fettigen Lobulierung („Wurst-Pankreas"), fokale Formen, kann simultane Befunde in anderen Organen aufweisen (z. B. Gallengänge, Speicheldrüsen, Aorta und retroperitoneales Fettgewebe)

MRCP: diffuse Einengung oder segmentale Stenose des Pankreashauptgangs („pancreatic duct penetrating sign“) ohne Gangaufstau, evtl. Strikturen des pankreatischen Segments des Ductus choledochus, proximaler Gallengangsaufstau und Gallenblasenvergrößerung

Bakterielle Pneumonie:

asymmetrische Konsolidierungen mit Luftbronchogramm, Pleuraerguss

Medikamenteninduzierte Pneumonitis:

Durchsicht der Medikamentenhistorie

Strahlenpneumonitis:

betrifft Lungenabschnitte die über einen Dosisschwellenwert von 30-40 Gy bestrahlt wurden, berücksichtigt keine anatomischen Grenzen wie interlobuläre Fissuren und bronchiovaskuläre Strukturen, zeigt Mattglasverschattungen, welche im zeitlichen Verlauf an Dichte zunehmen und schließlich konsolidieren

Hypophysenmakroadenom:

asymmetrische oder fokal vergrößerte Hypophyse mit normalem Stiel, heterogene Kontrastmittelaufnahme ohne durale Ausläufer Hypophysenmetastasen:

Melanom, Brust- und Lungenkarzinome (selten)

Autoimmunhypophysitis:

junge Frauen während der Schwangerschaft oder postnatalen Periode, Kopfschmerz, Visuseinschränkungen und ACTH-Mange

Rheumatoide Arthritis:

symmetrischer Befall kleiner Gelenke, Metakarpophalangeale und proximale Interphalangealgelenke, antizyklische Citrullinatedpeptid-Antikörper, Rheumafaktoren 


\begin{tabular}{l|l|l|l|l}
$\begin{array}{l}\text { Immunbedingte } \\
\text { Nebenwirkung }\end{array}$ & Typische bildgebende Befunde & Wichtige Differenzialdiagnosen \\
\hline $\begin{array}{l}\text { Sarkoidähnliche } \\
\text { Lymphadenopathie }\end{array}$ & $\begin{array}{l}\text { Symmetrische mediastinale und bihiläre Lymphknoten- } \\
\text { vergrößerung mit entweder neuen oder vergrößerten } \\
\text { vorbestehenden Lymphknoten }\end{array}$ & $\begin{array}{l}\text { Metastatische Lymphadenopathie: } \\
\text { oft asymmetrisch, evtl. inhomogenes Kontrastmittelverhalten mit } \\
\text { inhärenten oder behandlungsbedingten Nekrosen }\end{array}$ \\
\begin{tabular}{|l} 
MRCP Magnetresonanz-Cholangiopankreatikographie, PET Positronenemissionstomographie, FDG Fluordesoxyglukose, ACTH adrenokortikotropes \\
Hormon
\end{tabular}
\end{tabular}

tersuchung berichteten Kim et al. [17] vermehrte mesenteriale Gefäßzeichnung in $83 \%$, Darmwandverdickung in $75 \%$, und flüssigkeitsgefüllte distendierte Darmschlingen in $25 \%$ der Fälle. Perikolische Fettgewebsdichteerhöhungen wurden bei $16 \%$ der Patienten in einer Fallsammlung von Barina et al. [4] berichtet. Eine Pneumatose oder ein Pneumoperitoneum wurden nicht beobachtet $[4,17]$. Die immunbedingte Kolitis kann in 3 verschiedenen Mustern auftreten (• Tab. 1 und Beispiel in - Abb. 1; [4]):

- diffuse Kolitis,

- segmentale Kolitis assoziiert mit Divertikulitis und

- isolierte rektosigmoidale Kolitis ohne Divertikulose.

Bei segmentaler Kolitis assoziiert mit Divertikulitis waren die Wandverdickung des Kolons stärker und perikolische Fettgewebsdichteerhöhungen häufiger als bei diffuser Kolitis [17]. Klinische Symptome mit gemischt wässriger und blutiger Diarrhö und krampfartigen Schmerzen waren schwerer in der Gruppe mit segmentaler Kolitis assoziiert mit Divertikulitis; hingegen zeigte die Gruppe mit diffuser Kolitis und isolierter rektosigmoidaler Kolitis ohne Divertikulose leichtere Symptome mit vorwiegend wässriger Diarrhö $[4,17]$. Eine Ipilimumab-assoziierte Ileitis ohne Kolitis ist extrem selten und wurde in einem Fallbericht vorgestellt [29].

Wichtige Differenzialdiagnosen der immunbedingten Kolitis sind Morbus Crohn, Colitis ulcerosa sowie die infektiöse und pseudomembranöse Kolitis (- Tab. 1). Morbus Crohn betrifft vorwiegend das terminale Ileum und hat eine ungleichmäßige und transmurale Verteilung, die Colitis ulcerosa befällt typischerweise das Kolon mit zunehmender Intensität nach distal. Eine infektiöse Kolitis ist vorwiegend auf das rechte Kolon beschränkt, und die pseudomembranöse Kolitis zeigt eine sehr ausgeprägte zirkumferenzielle oder exzentrische Wandverdickung des gesamten Kolons [11].

\section{Hepatitis}

Immunbedingte hepatotoxische Nebenwirkungen, die zu einer Erhöhung der Transaminasen und ggf. des Bilirubins führen, treten in ungefähr $1-10 \%$ bei einer Monotherapie, aber in bis zu $30 \%$ unter der Kombinationstherapie Nivolumab plus Ipilimumab auf $[19,26]$. Eine Grad-3-Toxizität mit 3- bis 5-fach über dem oberen Normlimit erhöhten hepatischen Transaminasen wurde bei 1-2\% der Patienten in der Monotherapie und $14 \%$ der Patienten in der Kombinationstherapie beobachtet [26]. Die Patienten sind zumeist asymptomatisch, können aber Fieber, Müdigkeit, Gelbsucht und Stuhlverfärbungen aufweisen [11].

In der CT und MRT zeigt die manifeste immunbedingte Hepatitis eine Hepatomegalie, periportales Ödem, verminderte Leberdichte im Vergleich zur Baseline-CT, periportale T2-Signalsteigerung in der MRT und vergrößerte periportale Lymphknoten (• Tab. 1 und $\bullet$ Abb. 2; [16, 28]). Im Ultraschall (US) wurden ergänzend eine periportale Echogenitätssteigerung und ein Gallenblasenwandödem beschrieben $[11,16]$. In einer Fallserie mit 6 Patienten mit Ipilimumab-assoziierter Hepatitis berichteten Kim et al. [16], dass die Dichteminderung des Leberparenchyms Lebermetastasen maskieren und dass das Auftreten neuer landkartenartiger Areale mit niedriger Dichte $\mathrm{Me}$ tastasen ähneln kann. In Verlaufsuntersuchungen nach Glukokortikoidtherapie zeigt sich üblicherweise ein vollständiger Rückgang der Hepatomegalie und periportalen Lymphadenopathie [16].
Die bildgebenden Befunde der immunbedingten Hepatitis sind unspezifisch und ähneln denen einer viralen $\mathrm{He}$ patitis, alkoholinduzierten Hepatitis und Autoimmunhepatitis. Des Weiteren müssen differenzialdiagnostisch eine extrahepatische Cholestase und das Neuauftreten von Lebermetastasen ausgeschlossen werden [16].

\section{Pankreatitis}

Die immunbedingte Pankreatitis ist selten und zeigt eine Inzidenz von $<1 \%$ [28]. Sie geht mit einer Erhöhung der Serumamylase und Lipase einher, kann klinisch asymptomatisch sein oder Oberbauchschmerzen verursachen [19].

In der CT und MRT finden sich eine Organvergrößerung, Verminderung der Parenchymdichte und eine Dichteerhöhung des umgebenden Fettgewebes (- Tab. 1 und - Abb. 3; [28]). In der Positronenemissionstomographie(PET)CT zeigt die immunbedingte Pankreatitis eine gesteigerte Fluordesoxyglukose(FDG)-Aufnahme des Pankreas [20]. Es kann sehr schwierig sein, die Befunde von einer Immunoglobulin-G4assoziierten Autoimmunpankreatitis zu unterscheiden [20]. Im Unterschied zur immuntherapieinduzierten Pankreatitis kann die Immunoglobulin-G4-assoziierte Autoimmunpankreatitis jedoch auch fokale Formen aufweisen. Die typische Auflösung der normalen fettigen Lobulierung wird als „Wurst-Pankreas“ beschrieben, und es können simultane Befunde in anderen Organen auftreten (wie z. B. Gallengänge, Speicheldrüsen, Aorta und retroperitoneales Fettgewebe). Die Magnetresonanzcholangiopankreatographie (MRCP) kann eine diffuse Einengung oder segmentale Stenose des Pankreashauptgangs („pancreatic duct penetrating sign") ohne Gangaufstau zeigen oder Strikturen des pankreatischen 

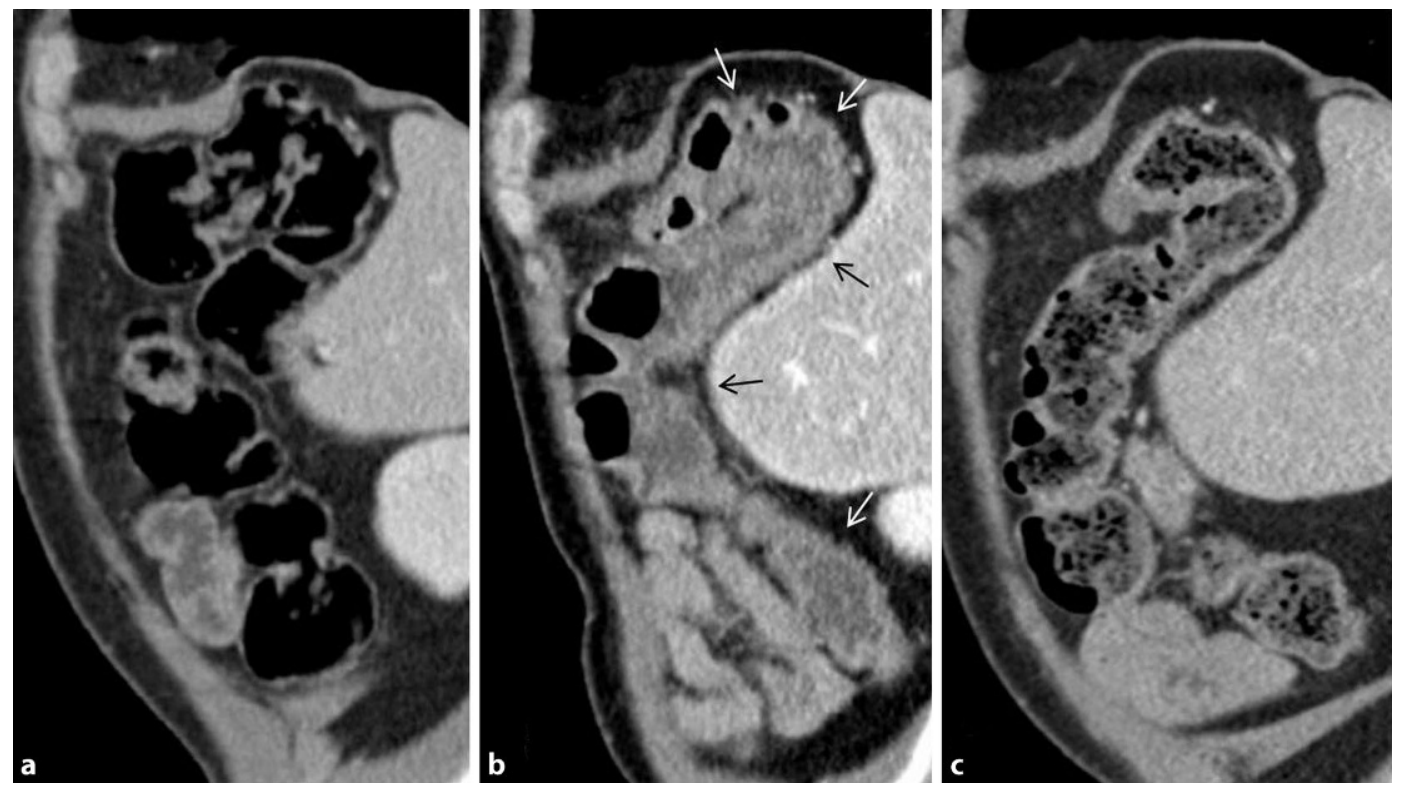

Abb. $1<72$ Jahre alter Mann mit Kolitis Grad 2 nach der 4. Gabe von Ipilimumab. a Normales KoIon vor Ipilimumab-Gabe. b Kolitis mit diffusem Kolitismuster. Wandverdickung des Kolons und vermehrte Kontrastmittelaufnahme der Mukosa (Pfeile). c Befundverbesserung nach Unterbrechung von Ipilimumab und Glukokortikoiden. (Copyright: G. Widmann)
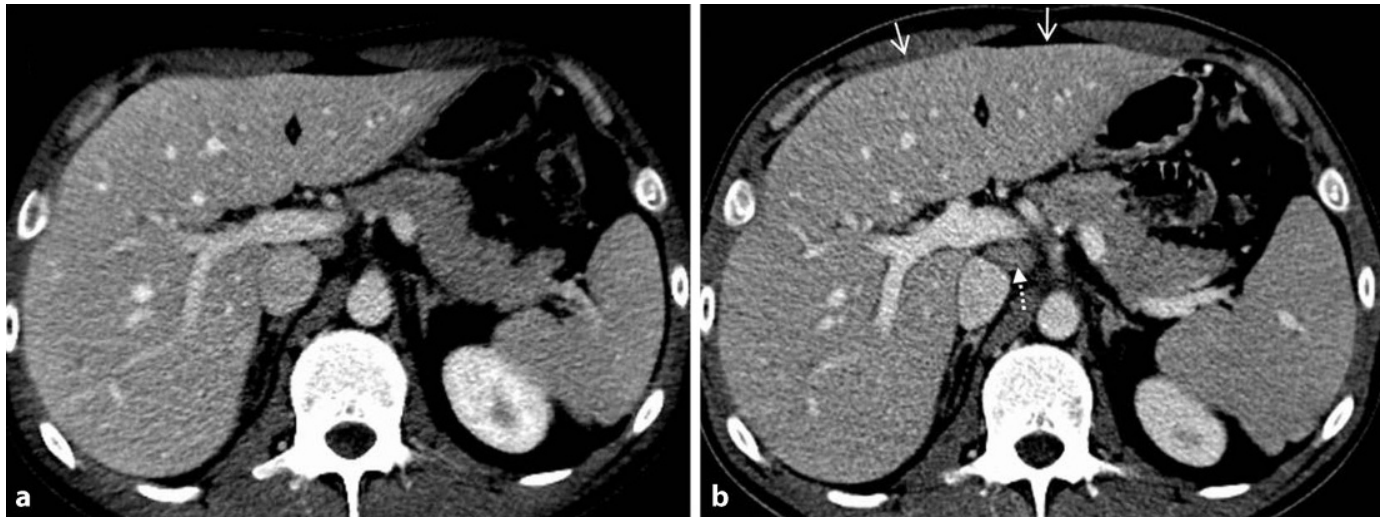

Abb. $2<33$ Jahre alter Mann mit Hepatitis Grad 3 nach der 3. Gabe von $\mathrm{Ni}$ volumab plus Ipilimumab. a Normal große Leber vor der Kombinationstherapie. b Hepatitis mit konvex geformter Hepatomegalie (Pfeile) und periportaler Lymphadenopathie (unterbrochener Pfeil). (Copyright: G. Widmann)

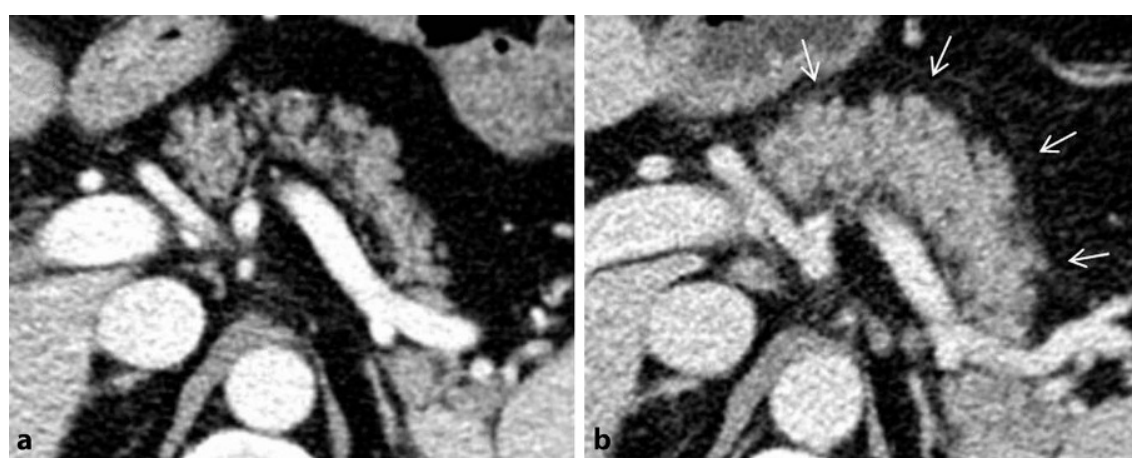

Abb. 3 A 54 Jahre alter Mann mit Pankreatitis nach der 1. Gabe von Pembrolizumab. a Normales Pankreas vor Pembrolizumab. b Pankreatitis mit Vergrößerung des Organs und geringer umgebender Fettgewebsdichtezunahme (Pfeile). (Copyright: G. Widmann)

Segments des Ductus choledochus, proximalen Gallengangsaufstau und Gallenblasenvergrößerung aufweisen. Veränderungen des Pankreashauptgangs und der Gallenwege im Rahmen der immuntherapieinduzierten Pankreatitis wurden bisher nicht berichtet. Im Unterschied zum zumeist milden klinischen Bild der immuntherapieinduzierten Pankreatitis präsentiert sich eine ImmunoglobulinG4-assoziierte Autoimmunpankreatitis häufig mit obstruktiver Gelbsucht und Oberbauchschmerzen.

\section{Pneumonitis}

Immunogene pulmonale Toxizitäten sind selten und treten typischerweise erst spät auf, ungefähr 10 Monate nach Beginn der Therapie [19]. Die Symptomatik reicht von beschwerdefrei und mild bei Grad 1 und 2 bis Husten, Hypoxie und lebensbedrohlichem Atemversagen mit Notwendigkeit der Hospitalisierung und intensivmedizinischen Maßnahmen bei Grad 3 und $4[15,19]$. Eine immunbedingte Pneumonitis wurde häufiger berichtet bei Patienten mit fortgeschrittenem Lungenkrebs (3-5\%) als für Patienten mit malignem Melanom (0-2\% [26]). Die Prävalenz ist höher für Nivolumab und Pembrolizumab und die Nivolumab-/Ipilimumab-Kombinationstherapie als für die IpilimumabMonotherapie [1, 18]. Patienten mit vorbestehender Lungenerkrankung und 


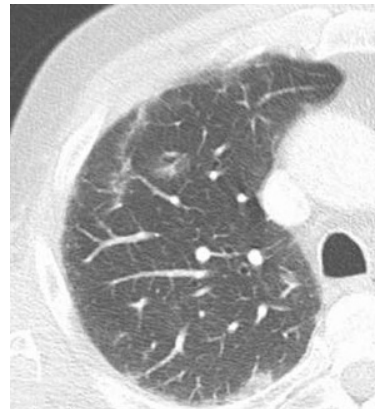

a

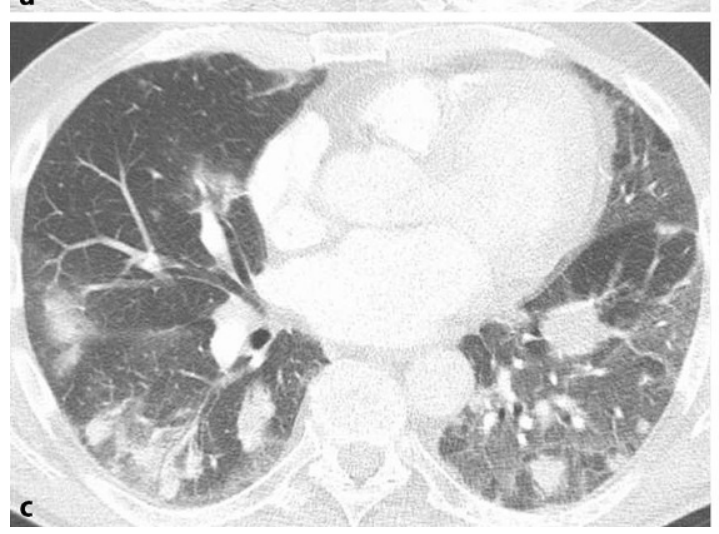

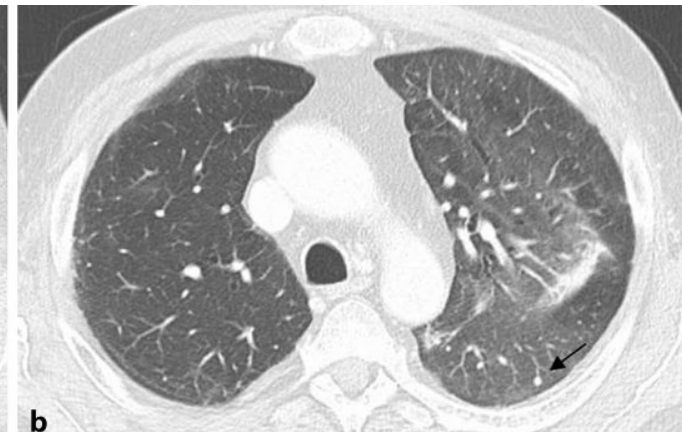

b

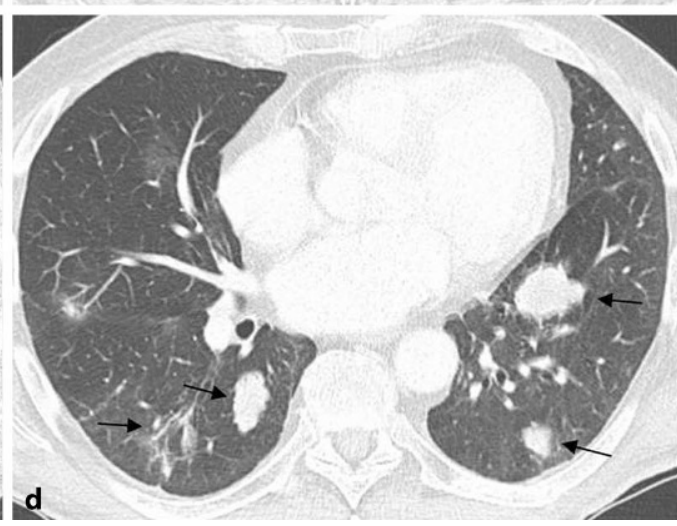

Abb. $4<73$ Jahre alter Mann mit Pneumonitis Grad 3 nach der 3. Gabe von Ipilimumab sequenziell nach der 9. Gabe von Pembrolizumab wegen Tumorprogression. $\mathbf{a}$, b Pneumonitis mit Muster der kryptogenen organisierenden Pneumonie. Gemischte multifokale periphere Mattglasverschattungen und Konsolidierungen. c, $d$ Befundverbesserung nach Absetzen von Ipilimumab und Glukokortikoiden. Die Pfeile in $\mathbf{b}$ und $\mathbf{d}$ zeigen multiple Lungenmetastasen. (Copyright: G. Widmann) erhaltener Radiotherapie von Lungenmetastasen vor Initiierung der Immuncheckpointinhibitortherapie scheinen ein erhöhtes Risiko für die Entwicklung einer immunbedingten Pneumonitis zu haben [23, 27].

Entsprechend der Klassifikation der American Thoracic Society/European Respiratory Society für interstitielle Pneumonien ordneten Nishino et al. [25] die radiologischen Befunde von 20 Patienten mit PD1-assoziierter Pneumonitis folgenden Mustern zu:

- kryptogene organisierende Pneumonie (COP) in $65 \%$,

- unspezifische interstitielle Pneumonie (NSIP) in $15 \%$,

- Hypersensitivitätspneumonitis (HP) in $10 \%$ und

- akute interstitielle Pneumonie/akutes respiratorisches Distresssyndrom (AIP/ARDS) in $10 \%$.

Die unteren Lungenabschnitte waren häufiger betroffen als die mittleren und oberen [25]. Gemischte und multifokale Verteilungen wurde häufiger beobachtet als periphere und basale Verteilungen [25]. Milchglasverdichtungen wurden in allen Fällen gefunden, retikuläre Muster und Konsolidierungen in den meisten
Fällen [25] - • Tab. 1 zeigt eine Zusammenfassung der Befunde und • Abb. 4 ein Beispiel mit COP-Muster. Nach dem Auslaufen der Glukokortikoidtherapie kann sich ein „flare“ der klinischen und bildgebenden Befunde einer rezidivierenden Pneumonitis zeigen [25].

Die Bildgebung kann helfen, andere pulmonale Erkrankungen abzugrenzen, wie z. B. eine bakterielle Pneumonie, die typischerweise asymmetrische Konsolidierungen mit positivem Pneumobronchogramm und eventuell einen Pleuraerguss aufweist [30]. Zusätzliche Informationen wie Befundpersistenz nach Antibiose sowie negative Kulturen aus Sputum, Bronchiallavage und Pleuraerguss können wichtige Hinweise auf eine immunbedingte Pneumonitis sein [30]. Zur Abgrenzung pulmonaler Toxizitäten von zytotoxischen und nicht zytotoxischen Medikamenten ist eine sorgfältige Durchsicht der Medikamentenhistorie notwendig. Eine Strahlenpneumonitis betrifft Lungenabschnitte, die mit mehr als 30-40 Gy belastet wurden und respektiert keine anatomischen Grenzen wie interlobuläre Fissuren und bronchiovaskuläre Strukturen [13]. Typischerweise beginnt sie nach 6 bis 10 Wochen und zeigt Milchglasverschat- tungen, die an Dichte zunehmen und schließlich konsolidieren [13].

\section{Hypophysitis}

Die Hypophysitis wurde als eine Komplikation der Ipilimumab-Therapie beschrieben [19]. Sie wird synonym als Ipilimumab-induzierte Hypophysitis (IIH), oder Ipilimumab-assoziierte Autoimmunhypophysitis (IAH) bezeichnet [3]. Die Hypophysitis tritt gewöhnlich nach dem 3. Zyklus von Ipilimumab auf, also etwa 9 Wochen nach Therapiebeginn und hat eine Inzidenz von 2-4\% [19]. Höhere Inzidenzen von 8 \% wurden von Larkin et al. [22] für die Kombinationstherapie Nivolumab plus Ipilimumab und bis zu $25 \%$ von Albarel et al. [2] für die hoch dosierte Therapie mit Ipilimumab (10 mg/kgKG) beschrieben. Initiale Symptome sind Kopfschmerzen und Müdigkeit, gefolgt von einer Insuffizienz der thyreo-, gonado- und kortikotropen Achse, die auch schwerwiegend bis lebensbedrohlich sein kann [2, 3, 19].

In der bildgebenden Übersichtsarbeit von Araujo et al. [3] über 57 Fälle von Ipilimumab-assoziierter Hypophysitis konnten pathologische Befunde der Hypophyse in $77 \%$ nachgewiesen 

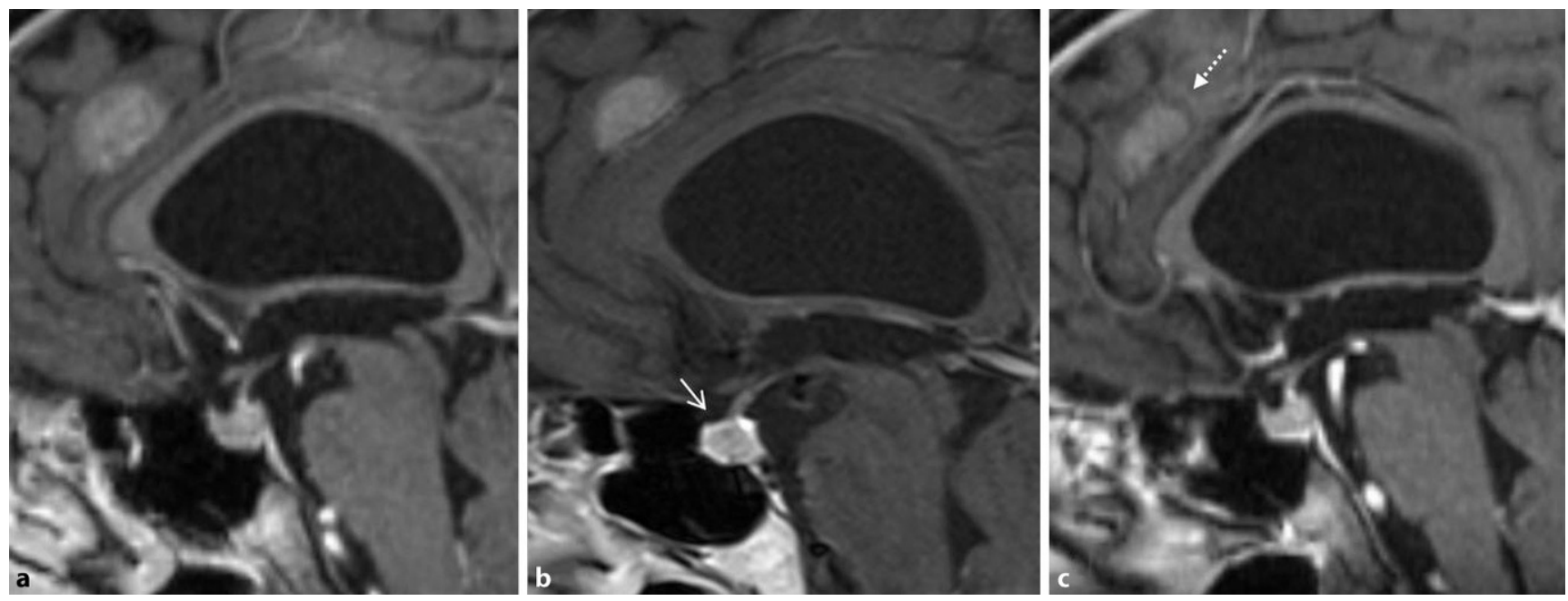

Abb. 5 \& 73 Jahre alter Mann mit Hypophysitis Grad 3 nach der 5. Gabe von Ipilimumab. a Normale Hypophyse vor Ipilimumab-Gabe. b Hypophysitis mit moderater Vergrößerung der Hypophyse und homogener Kontrastmittelaufnahme (Pfeil). c Rückgang der Hypophyse zur normalen Größe nach Absetzen von Ipilimumab und Glukokortikoiden. UnterbrochenerPfeil: Hirnmetastase mit partieller Regression. (Copyright: G. Widmann)
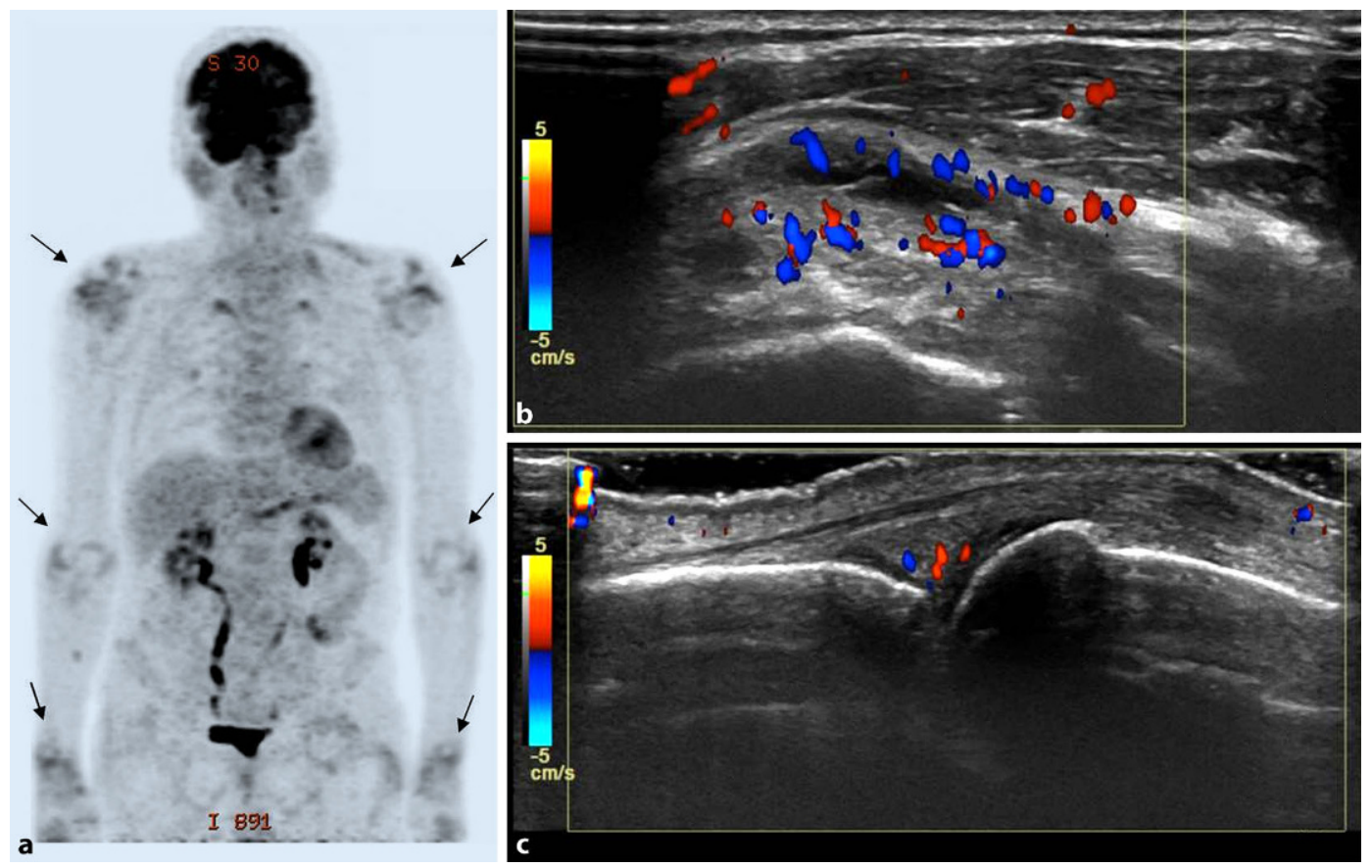

Abb. $6<76$ Jahre alter Mann mit Arthritis nach der 21. Gabe von Nivolumab. a PET-CT: Oligoarthritis mit vermehrter FDG-Anreicherung in den Schultern, Ellenbögen und Handgelenken (Pfeile). b Schultergelenkarthritis mit verdickter Synovia und vermehrter Durchblutung. c Metakarpophalangealgelenkarthritis mit verdickter hypervas kularisierter Synovia. PET Positronenemissionstomographie, FDG Fluordesoxyglukose. (Copyright: G. Widmann)

werden. Typische MRT-Befunde sind eine moderate Vergrößerung der Hypophyse, konvexe obere Kontur, Vergrößerung des Stiels oder Infundibulums und eine homogene Kontrastmittelaufnahme (-Tab. 1 und Abb. 5; [2, 3]). Eine heterogene Kontrastmittelaufnahme wurde als ein untypisches Muster beschrieben [10]. Marlier et al. [24] fanden einer Vergrößerung der Hypophyse in jenen 2 Fällen, in denen die Dosis von Ipilimumab $10 \mathrm{mg} / \mathrm{kgKG}$ betragen hatte, während die übrigen 2 symptomatischen Patienten, die $3 \mathrm{mg} / \mathrm{kgKG}$ Ipilimumab erhalten hatten, eine normal große Hypophyse aufwiesen.

MRT-Kontrollen im Verlauf nach Glukokortikoidbehandlung können eine Abnahme des Hypophysenvolumens, Rückgang der konvexen in eine konkave Kontur oder im Extremfall eine „leere Sella“ zeigen [2]. Bei der Mehrzahl der betroffenen Patienten (64-76\%) normalisiert sich die Hypohphysenfunk- tion nicht $[3,14]$. Eine immunbedingte Thyreoiditis, die gewöhnlich in einer Hypothyreose mündet, kann entweder isoliert oder gemeinsam mit einer Hypophysitis auftreten und zu einer erhöhten FDG-Aufnahme in der PET-CT führen [28].

Wichtige Differenzialdiagnosen der Ipilimumab-assoziierten Hypophysitis sind Hypophysenmakroadenome und seltene Fälle von Hypophysenmetastasen durch Melanome, Brust- und 

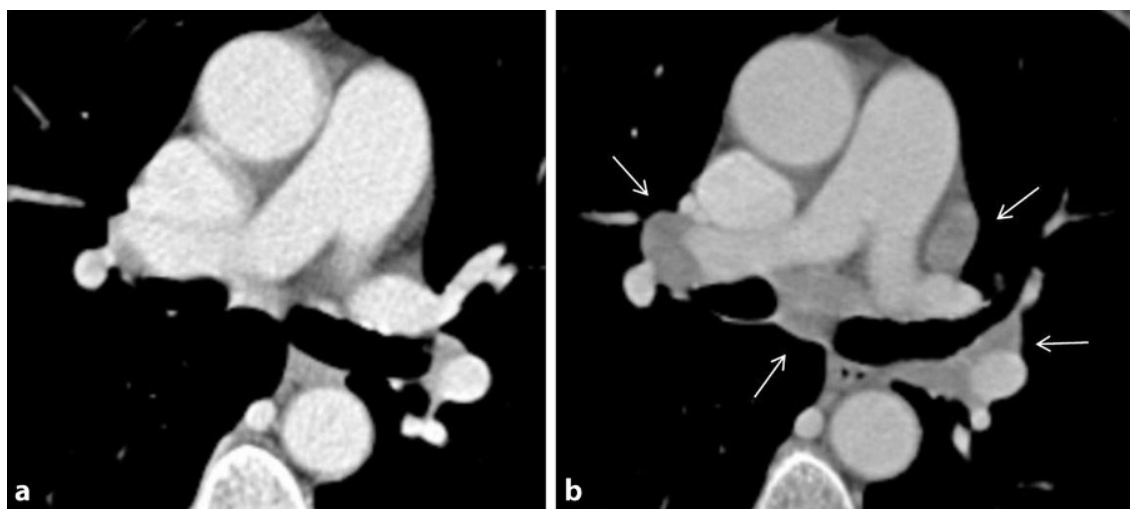

Abb. 7 ॥ 67 Jahre alte Frau mit sarkoidähnlicher Lymphadenopathie nach der 9. Gabe von Pembrolizumab. a Normale mediastinale und hiläre Lymphknoten vor Pembrolizumab-Gabe. b Sarkoidähnliche Lymphadenopathie (histologisch verifiziert) mit symmetrischer hilärer und mediastinaler Lymphknotenvergrößerung (Pfeile). (Copyright: G. Widmann)

Lungenkarzinome [3]. Im Gegensatz zur Hypophysitis gehen Makroadenome gewöhnlich mit einer asymmetrischen oder fokal vergrößerten Hypophyse mit normalem Stiel einher und zeigen eine heterogene Kontrastmittelaufnahme ohne durale Ausläufer [10]. Autoimmunerkrankungen der Hypophyse treten sonst vorwiegend bei jungen Frauen während der Schwangerschaft oder postnatalen Periode auf und präsentieren sich klinisch mit Kopfschmerz, Visuseinschränkungen und ACTH-Mangel (adrenokortikotropes Hormon).

\section{Arthritis}

Rheumatologische Nebenwirkungen der Immuncheckpointinhibitortherapie beinhalten Arthralgien, Myalgien, Arthritis und Myositis [18]. Die Inzidenz von Arthralgien liegt zwischen 9-12 \% und 6-8 \% unter Pembrolizumab und Nivolumab, $5 \%$ unter Ipilimumab und $11 \%$ unter der Kombinationstherapie von Nivolumab plus Ipilimumab [26]. Die manifeste Arthritis ist weniger häufig (Prävalenz ca. $2 \%$ unter Anti-PD-1-Inhibitoren [26, 27]). In einer Fallserie von Cappelli et al. [7] mit 13 Patienten mit immunbedingten rheumatologischen Nebenwirkungen, lag in 9 Fällen eine Arthritis vor, in 4 Fällen eine bildgebend bestätigte Synovitis mit entzündlicher Synovialflüssigkeit. Die Arthritis kann sowohl große als auch kleine Gelenke betreffen und sich als Oligoarthritis, additive Arthritis oder schwere Polyarthritis manifestieren [7, 26, 27].

Befunde im US und MRT sind eine proliferative Synovitis mit typischer Hyperämie und synovialer Verdickung, ein Gelenkerguss, manchmal Gelenkerosionen, Tendosynovitis, Knochenmarködem und Myositis (ब Tab. 1 und - Abb. 6; $[7,8]$ ). In der PET-CT zeigt sich eine erhöhte FDG-Aufnahme in der Synovia multipler bilateraler Gelenke bei Arthritis und bei Myositis in Muskeln (• Abb. 6; [6, 20]). Eine Differenzierung zur rheumatoiden Arthritis kann sehr schwierig sein, antizyklische Citrullinated-peptid-Antikörper und Rheumafaktoren werden jedoch für gewöhnlich nicht gefunden [7]. Suarez-Almazor et al. [27] postulierten 2 potenzielle Erklärungsmodelle für immunbedingte Arthritiden: Eine unspezifische Arthritis infolge einer Hochregulierung des Immunsystems und eine rheumatoide Arthritis auf dem Boden einer genetischen oder umweltbedingten Prädisposition, die durch die Immuncheckpointinhibition getriggert wird.

\section{Sarkoidähnliche Lymphadenopathie}

Eine immunbedingte sarkoidähnliche Lymphadenopathie (engl. „sarcoid-like reaction") wurde als asymptomatischer radiologischer Befund bei ca. 5-7\% der Fälle beschrieben $[5,6,28]$. Sie kann sich als symmetrische mediastinale und bihiläre Lymphknotenvergrößerung ma- nifestieren, mit entweder neuen oder vergrößerten vorbestehenden Lymphknoten (•Tab. 1 und $\bullet$ Abb. 7; [6]). Tirumani et al. [28] berichteten über gleichzeitige pulmonale Befunde wie bilaterale irreguläre noduläre und fleckige Verdichtungen oder Milchglasverschattungen und interstitielle Verdichtungen bei 3 von 8 Patienten. Die immunbedingte sarkoidähnliche Lymphadenopathie kann in den meisten Fällen wieder abklingen [6, 28].

Eine Differenzierung der immunbedingten sarkoidähnlichen Lymphadenopathie von metastatischen und reaktiven Lymphknoten kann sehr schwierig sein, da die bildgebenden Befunde unspezifisch und fehlleitend sein können [5]. Metastatische Lymphknoten können ein inhomogenes Kontrastmittelverhalten aufweisen und inhärente oder behandlungsbedingten Nekrosen entwickeln [6].

\section{Nebenwirkungen neuerer Immuncheckpointinhibitoren}

Die bildgebenden Befunde der toxischen Effekte neuerer Immuncheckpointinhibitoren wie Atezolizumab, Tremelimumab und Pidilizumab wurden in dieser Übersicht wegen der geringen Datenlage nicht erfasst, dürften sich aber aufgrund des ähnlichen Wirkmechanismus gleichen.

\section{Fazit für die Praxis}

- Die zunehmende Verwendung von Immuncheckpointinhibitoren erhöht die Notwendigkeit radiologischer Kompetenzen zur Abklärung immunbedingter Nebenwirkungen.

- Die wichtigsten bildgebenden Manifestationen sind Kolitis, Hepatitis, Pankreatitis, Hypophysitis, Pneumonitis, Arthritis und die sarkoidähnliche Lymphadenopathie; lebensbedrohliche Komplikationen können aus einer Kolitis, Pneumonitis und Hypophysitis resultieren.

- Die CT ist für die Abklärung akuter gastrointestinaler und pulmonaler Nebenwirkungen die Methode der Wahl, MRT für die Hypophysitis und US für rheumatologische Nebenwirkungen. PET-CT-Untersuchungen 


\section{zeigen einen erhöhten Glukosestoff- wechsel der betroffenen Organe. \\ - Die Abgrenzung von anderen Au- toimmunerkrankungen sowie einer immunbedingten Pneumonitis von entzündlichen oder medikamenten- bedingten pulmonalen Erkrankun- gen kann sehr schwierig sein und bedarf einer engen multidiszipli- nären Zusammenarbeit.}

\section{Korrespondenzadresse}

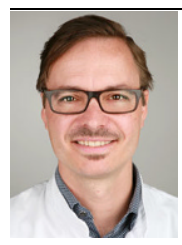

PD Dr. G. Widmann

Universitätsklinik für Radiologie, Medizinische Universität Innsbruck

Anichstr. 35, 6020 Innsbruck, Österreich gerlig.widmann@i-med.ac.at

Acknowledgements. Open access funding provided by University of Innsbruck and Medical University of Innsbruck.

\section{Einhaltung ethischer Richtlinien}

Interessenkonflikt. G. Widmann, V.A. Nguyen, J. Plaickner und W. Jaschke geben an, dass kein Interessenkonflikt besteht.

Dieser Beitrag beinhaltet keine von den Autoren durchgeführten Studien an Menschen oder Tieren.

Open Access. Dieser Artikel wird unter der Creative Commons Namensnennung 4.0 International Lizenz (http://creativecommons.org/licenses/by/4.0/deed. de) veröffentlicht, welche die Nutzung, Vervielfältigung, Bearbeitung, Verbreitung und Wiedergabe in jeglichem Medium und Format erlaubt, sofern Sie den/die ursprünglichen Autor(en) und die Quelle ordnungsgemäßnennen, einen Linkzur Creative Commons Lizenz beifügen und angeben, ob Änderungen vorgenommen wurden.

\section{Literatur}

1. Abdel-Rahman O, Fouad M (2016) Risk of pneumonitis in cancer patients treated with immune checkpoint inhibitors: a meta-analysis. Ther Adv Respir Dis 10:183-193

2. Albarel F, Gaudy C, Castinetti $F$ et al (2015) Long-term follow-up of ipilimumab-induced hypophysitis, a common adverse event of the antiCTLA-4 antibody in melanoma. Eur J Endocrinol 172:195-204

3. Araujo PB, Coelho MC, Arruda M et al (2015) Ipilimumab-induced hypophysitis: review of the literature. J Endocrinol Invest 38(11):1159-1166. doi:10.1007/s40618-015-0301-z

4. Barina AR, Bashir MR, Howard BA et al (2016) Isolated recto-sigmoid colitis: a new imaging pattern of ipilimumab-associated colitis. Abdom
Radiol (NY) 41(2):207-214. doi:10.1007/s00261015-0560-3

5. Braschi-Amirfarzan M, Tirumani SH, Hodi FS Jr. et al (2017) Immune-checkpoint inhibitors in the era of precision medicine: What radiologists should know. Korean J Radiol 18:42-53

6. Bronstein Y, Ng CS, Hwu P et al (2011) Radiologic manifestations of immune-related adverse events in patients with metastatic melanoma undergoing anti-CTLA-4 antibody therapy. AJR Am J Roentgenol 197:W992-W1000. doi:10.2214/ajr. 10.6198

7. Cappelli LC, Gutierrez AK, Baer AN et al (2017) Inflammatoryarthritis and sicca syndromeinduced by nivolumab and ipilimumab. Ann Rheum Dis 76(1):43-50. doi:10.1136/annrheumdis-2016209595

8. Chan MM, Kefford RF, Carlino M et al (2015) Arthritis and tenosynovitis associated with the anti-PD1 antibody pembrolizumab in metastatic melanoma. JImmunother 38:37-39

9. Cheng R, Cooper A, Kench Jetal (2015) Ipilimumabinduced toxicities and the gastroenterologist. J Gastroenterol Hepatol 30:657-666

10. Chodakiewitz Y, Brown S, Boxerman JL et al (2014) Ipilimumab treatment associated pituitary hypophysitis: clinical presentation and imaging diagnosis. Clin Neurol Neurosurg 125:125-130. doi:10.1016/j.clineuro.2014.06.011

11. Cramer P, Bresalier RS (2017) Gastrointestinal and hepatic complications of immune checkpoint inhibitors. Curr Gastroenterol Rep 19(1):3. doi:10. 1007/s11894-017-0540-6

12. Demlova R, Valik D, Obermannova R et al (2016) The safety of therapeutic monoclonal antibodies: implicationsforcancer therapyincluding immunocheckpoint inhibitors. Physiol Res 65:S455-S462

13. Diederich S (2016) Chest CT for suspected pulmonary complications of oncologic therapies: How I review and report. Cancer Imaging 16:7

14. Faje AT, Sullivan R, Lawrence D et al (2014) Ipilimumab-induced hypophysitis: a detailed longitudinal analysis in a large cohort of patients with metastatic melanoma. J Clin Endocrinol Metab 99(11):4078-4085. doi:10.1210/jc.20142306

15. Heinzerling L, Goldinger SM (2017) A review of serious adverse effects under treatment with checkpointinhibitors. Curr Opin Oncol 29:136-144

16. Kim KW, Ramaiya NH, Krajewski KM et al (2013) Ipilimumab associated hepatitis: imaging and clinicopathologic findings. Invest New Drugs 31(4):1071-1077. doi:10.1007/s10637-013-99396

17. Kim KW, Ramaiya NH, Krajewski KM et al (2013) Ipilimumab-associated colitis: CT findings. AJR Am J Roentgenol 200(5):W468-W474. doi:10.2214/ajr. 12.9751

18. Kourie HR, Klastersky J (2016) Immune checkpoint inhibitors side effects and management. Immunotherapy 8(7):799-807. doi:10.2217/imt-20160029

19. Kumar V, Chaudhary N, Garg M et al (2017) Current diagnosis and management of immune related adverse events (irAes) induced by immune checkpoint inhibitor therapy. Front Pharmacol 8:49

20. Kwak JJ, Tirumani SH, Van Den Abbeele AD et al (2015) Cancer immunotherapy: imaging assessment of novel treatment response patterns and immune-related adverse events. Radiographics 35(2):424-437. doi:10.1148/rg.352140121

21. La-Beck NM, Jean GW, Huynh C et al (2015) Immune checkpoint inhibitors: new insights and current place in cancer therapy. Pharmacotherapy 35(10):963-976. doi:10.1002/phar.1643

22. Larkin J, Hodi FS, Wolchok JD (2015) Combined nivolumab and ipilimumab or monotherapy in untreated melanoma. N Engl J Med 373(13):1270-1271. doi:10.1056/nejmc1509660

23. Lu CS, Liu JH (2017) Pneumonitis in cancer patients receiving anti-PD-1 and radiotherapies: three case reports. Medicine (Baltimore) 96(1):e5747. doi:10. 1097/md.0000000000005747

24. Marlier J, Cocquyt V, Brochez L et al (2014) Ipilimumab, not just another anti-cancer therapy: hypophysitis as side effect illustrated by four casereports. Endocrine 47:878-883

25. Nishino M, Ramaiya NH, Awad MMetal (2016) PD-1 inhibitor-related pneumonitis in advanced cancer patients: radiographic patterns and clinical course Clin Cancer Res 22(24):6051-6060. doi:10.1158/ 1078-0432.ccr-16-1320

26. Spain L, Diem S, Larkin J (2016) Management of toxicities of immune checkpoint inhibitors. Cancer Treat Rev 44:51-60

27. Suarez-Almazor ME, Kim ST, Abdel-Wahab N et al (2017) Review: immune-related adverse events with use of checkpoint inhibitors for immunotherapy of cancer. Arthritis Rheumatol 69(4):687-699. doi:10.1002/art.40043

28. Tirumani SH, Ramaiya NH, Keraliya A et al (2015) Radiographic profiling of immune-related adverse events in advanced melanoma patients treated with ipilimumab. Cancer Immunol Res 3(10):1185-1192. doi:10.1158/2326-6066.cir-150102

29. Venditti O, De Lisi D, Caricato M et al (2015) Ipilimumab and immune-mediated adverse events: a case report of anti-CTLA4 induced ileitis. BMC Cancer 15:87

30. Watanabe $\mathrm{S}$, Kimura $\mathrm{H}$, Takato $\mathrm{H}$ et al (2016) Severe pneumonitis after nivolumab treatment in a patient with melanoma. Allergol Int 65:487-489 